\title{
Activated TAZ Induces Liver Cancer in Collaboration with EGFR/HER2 Signaling Pathways
}

Hyuk Moon ( $\nabla$ hmoon@khu.ac.kr)

Kyung Hee University https://orcid.org/0000-0002-6252-2571

\section{Hye Jin Choi}

Yonsei University College of Medicine

\section{Do Young Kim}

Yonsei University College of Medicine

\section{Simon Weonsang Ro}

Kyung Hee University

\section{Research Article}

Keywords: Hepatocellular Carcinoma, Cholangiocarcinoma, TAZ, Hydrodynamic Transfection, EGFR/HER2

Posted Date: August 17th, 2021

DOl: https://doi.org/10.21203/rs.3.rs-796723/v1

License: (c) (1) This work is licensed under a Creative Commons Attribution 4.0 International License. Read Full License

Version of Record: A version of this preprint was published at BMC Cancer on April 19th, 2022. See the published version at https://doi.org/10.1186/s12885-022-09516-1. 


\section{Abstract}

Liver cancer is a major global health concern due to the steady increases in its incidence and mortality. Transcription factors, yes-associated protein (YAP) and WW domain-containing transcription regulator protein 1 (WWTR1, also known as TAZ)have emerged as critical regulators in human hepatocellular carcinoma (HCC) and cholangiocarcinoma (CC), the two major types of primary liver cancer. However, our study as well as other previous reports have shown that activation of YAP and TAZ (YAP/TAZ) in adult murine livers is insufficient for the development of liver cancer, suggesting a requirement for an additional oncogenic collaborator for liver carcinogenesis in adulthood. Therefore,we sought to identifythe oncogenic partners of YAP/TAZ that promote hepatocarcinogenesis in adults. Through database analyses, we identified EGFR/HER2signaling to be essential in human cancerswith high TAZ activity.Furthermore,immunohistochemical analyses showedthat human HCC and CC tissues with high YAP/TAZ activities exhibited concomitant activation of EGFR/HER2downstream signaling pathways. To demonstrate that EGFR/HER2 signaling promotes YAP/TAZ-mediated hepatocarcinogenesis, a constitutively active form of TAZ was simultaneously expressed in murine adult livers with effector molecules downstream of EGFR/HER2 signaling pathways.Interestingly, activated TAZ and BRAFinduced $\mathrm{HCC}$, whereas activated TAZ and PI3K led to the development of CC-like cancer, demonstrating that TAZ collaborates with EGFR/HER2 signaling pathways to induce both HCC and CC.

\section{Introduction}

Liver cancer is the fourth most common cancer worldwide, and its incidence and mortality rates have been increasing steadily (Jung et al. 2011; Villanueva 2019). Liver cancer typically exhibits heterogeneous histological features and poor prognosis (D. Y. Kim \& Han 2018; Llovet et al. 2016). The most common type of primary liver cancer is hepatocellular carcinoma (HCC), which accounts for about $80 \%$ of the cases, followed by cholangiocarcinoma (CC), which contributes to $10-20 \%$ of primary liver cancers (Hyder et al. 2014; Villanueva 2019). Although the two types of liver cancers have fundamentally different molecular and clinical characteristics, they share overlapping risk factors and oncogenic signaling pathways. Moreover, adult hepatocytes have been identified as the origin of both HCC and CC in recent studies (Y. Chen et al. 2012; Sia et al. 2017; Tanimizu et al. 2013).

The Hippo signaling pathway consists of a kinase cascade that regulates a variety of cellular processes (K. Harvey \& Tapon 2007; K. F. Harvey et al. 2013; Pan 2007). The core kinases include the mammalian STE20-like protein kinase 1 and 2 (MST1 and MST2, also known as STK4 and STK3, respectively), that phosphorylate the large tumor suppressor 1 (LATS1) and 2 (LATS2). When Hippo signaling is activated, LATS1 and LATS2 (LATS1/2) phosphorylate yes-associated protein (YAP) and WW domain-containing transcription regulator protein 1 (WWTR1, also known as TAZ), thereby inhibiting the nuclear import of YAP and TAZ (YAP/TAZ). In contrast, when Hippo signaling is inactivated, dephosphorylated YAP/TAZ is transported into the nucleus resulting in the transcriptional activation of a plethora of genes involved in cell proliferation and survival through interaction with the TEA domain family members (TEADs)(Mo et al. 2014; Moroishi et al. 2015; Zanconato et al. 2016). Recent years have witnessed significant advances in 
our understanding of the roles of YAP/TAZ in HCC and CC (Sugiura et al. 2019; Xu et al. 2009; Zender et al. 2006). Knockouts of the key mediators of the Hippo signaling pathway in embryos developed HCC and CC in adult mice (Hong et al. 2019; Lu et al. 2010; Song et al. 2010; N. Zhang et al. 2010). Likewise, prolonged overexpression of YAP/TAZ from neonatal stages induced HCC later in adulthood (Dong et al. 2007; Liu-Chittenden et al. 2012). In contrast to the prolonged activation of YAP/TAZ from embryonic stages or starting at birth, activation of YAP in the adult liver generally failed to induce cancer, but caused hepatomegaly or preneoplastic lesions (Dong et al. 2007; Yimlamai et al. 2014). This suggests that activation of YAP or TAZ alone, is insufficient to induce cancer in adult livers, and requires additional oncogenic partners. Liver cancers often show dysregulation of multiple signaling pathways such as RASRAF-MEK-ERK, PI3K-AKT-mTOR, Wnt/ $\beta$-catenin, and SHH signaling pathways (C. Chen \& Wang 2015; Yim \& Lee 2019). Concurrent alterations in multiple signaling pathways in liver cancer suggests that oncogenic collaborations may be required to initiate or promote tumor development in the liver. In this study, we used database analyses and a murine liver-specific transgenic model to identify the oncogenic partners of TAZ that promote tumorigenesis in the liver.

\section{Materials And Methods}

\section{Genomic data analyses from publicly available databases}

Data analysis of the transcriptome of patients with liver cancer was performed using the following publicly available databases: National center for biotechnology information (NCBI) gene expression omnibus (GEO) database (Accession Nos. GSE36376, GSE26566, GSE64041, GSE32958, GSE54236, and GSE32225) and the cancer genome atlas (TCGA) projects TCGA-LIHC (liver hepatocellular carcinoma) and TCGA-CHOL (cholangiocarcinoma).

\section{Statistical analysis}

Statistical analyses were carried out with two-tailed unpaired $t$-tests using GraphPad Prism Software (GraphPad, La Jolla, CA, USA). All values are expressed as means. Significant differences between two groups are denoted by asterisks $(* * *, P<0.001)$.

\section{Gsea}

Gene sets were downloaded from the molecular signatures database (MSigDB) (http://software.broadinstitute.org/gsea/) of the Broad Institute (Cambridge, MA, USA). Using the gene set permutation, the signal-to-noise ratio of the genes was used to determine the statistical enrichment of the gene sets associated with YAP/TAZ, MAPK, and AKT signaling pathways.

\section{Animal experiments}


Wild-type (C57BL6/N) male mice were purchased from Orientbio (Seongnam, Korea), maintained in a specific pathogen free (SPF) facility under a $12 \mathrm{~h}$ light/dark cycle, and provided food and water ad libitum. All experiments using mice were approved by the Institutional Animal Policy and Welfare Committee. Mice were randomly assigned to a hydrodynamic tail vein injection. For hydrodynamic tail vein injection, DNA mixtures containing transposons and transposase-encoding plasmids were prepared using the Endo-Free Maxi Kit (Qiagen, Hilden, Germany). The DNA plasmids were diluted in lactated Ringer's solution, and then injected into the lateral tail veins of male mice ( $5-6$ weeks old; $0.1 \mathrm{~mL} / \mathrm{g}$ body weight) within 7 s, as previously described (Ju et al. 2013).

\section{Recombinant DNA}

Transposons encoding the constitutively activated forms of YAP (pT3/EF5a YAPS127A) and TAZ (pT3/EF5a TAZS89A) were kind gifts from Dr. Xin Chen at the University of California, San Francisco. The plasmids pT2/EGFP and pPGK-SB13 were described previously (Moon et al. 2017). Open reading frames (ORFs) encoding the activated forms of BRAF (BRAF ${ }^{\mathrm{V} 600 \mathrm{E}}$ ) and PIK3CA (PI3K ${ }^{\mathrm{E} 545 \mathrm{~K}}$ ) were polymerase chain reaction (PCR)-amplified from pBabe-B-RAF-V600E (\#17544; Addgene, Watertown, MA, USA) and pBabe-puro-HA-PIK3CA-E545K plasmids (\#12525; Addgene), respectively. These amplified PCR products were cloned into pT2/BH transposon vectors (\#26556; Addgene) to generate the plasmids, pT2/ $\mathrm{BRAF}^{\mathrm{V} 600 \mathrm{E}}$, and $\mathrm{pT} 2 / \mathrm{PI} 3 \mathrm{~K}^{\mathrm{E} 545 \mathrm{~K}}$, respectively.

\section{CCL sensitivity analysis for compounds}

CTRP (v2) (http://www.broadinstitute.org/ctrp.v2.2) was used to investigate the correlation between sensitivity to chemicals and the copy number of TAZ in the CCLs. The CTRP v2 contains 860 CCLs, of which 827 are characterized as part of the Cancer Cell Line Encyclopedia, and represent 25 different lineages. The data included a $72 \mathrm{~h}, 16$-point dose-response screen to assess the sensitivity of these cell lines to a total of 481 compounds, including 70 FDA-approved drugs, 100 candidate compounds, and 311 small molecules (Rees et al. 2016; Seashore-Ludlow et al. 2015).

\section{Histology and IHC}

Liver tissue samples were fixed in 10\% neutral-buffered formalin and embedded in paraffin. The paraffin sections were deparaffinized in xylene and rehydrated by passing through gradually decreasing strengths of ethanol. The sections were then stained with hematoxylin and eosin (H\&E) and standard IHC for histopathological analysis. IHC staining for YAP/TAZ, phospho-MEK, phospho-AKT, and CK19 was conducted using antibodies against anti-YAP/TAZ (1:100; \#8418; Cell Signaling Technology, Danvers, MA, USA), anti-MEK1/2 (phospho-Ser217/221; 1:200; \#9154; Cell Signaling Technology), anti-AKT1 (PhosphoSer473; 1:400; ab81283; Abcam, Cambridge, UK), and anti-cytokeratin 19 (1:1000; ab133496; Abcam). After incubation with the primary antibodies, the sections were incubated with the appropriate biotinylated secondary antibodies, followed by treatment with freshly prepared DAB substrates (Vector Laboratories, Burlingame, CA, USA).

\section{Protein extraction and western blotting}


Frozen mouse liver samples were homogenized and digested in 1× RIPA lysis buffer containing complete protease and phosphatase inhibitor cocktails (P3200; GenDEPOT, Barker, TX, USA). Proteins were separated by sodium dodecyl sulfate polyacrylamide gel electrophoresis (SDS-PAGE) and transferred onto a polyvinylidene difluoride (PVDF) membrane. The membranes were immunoblotted with antibodies against phospho-AKT (\#4060; Cell Signaling Technology), phospho-MEK1/2 (\#9154; Cell Signaling Technology), NFkB p65 (sc-372; Santa Cruz Biotechnology, Dallas, TX, USA), Notch1 (ab8925; Abcam), and GAPDH (\#2118; Cell Signaling Technology). Finally, the immunoreactive proteins were detected using the West-Q Pico Dura ECL Solution (W3653; GenDEPOT, Barker, TX, USA).

\section{Human liver tissue specimens}

Human primary HCC and CC tissues were obtained from the biobank at the Severance Hospital, Seoul, Korea. Tissues were collected immediately following surgery and stored at $-80^{\circ} \mathrm{C}$ until processing and use. This study was approved by the Independent Institutional Review Board of Severance Hospital (IRB number: 4-2018-1087) and conformed to the ethical guidelines of the Declaration of Helsinki (1975).

\section{Results}

\section{Activation of YAP/TAZ signaling in human liver cancer}

First, we investigated whether YAP/TAZ activity was elevated in human liver cancer using publicly available databases. Expression levels of YAP and TAZ were significantly higher both in HCC and CC when compared to that in matching non-tumor hepatic tissues $(P<0.001$, Fig. $1 \mathrm{~A}$ and $\mathrm{B})$. Further, gene set enrichment analysis (GSEA) showed that YAP-conserved signature gene sets were highly enriched in both HCC and CC (Fig. 1C and Supplementary Table 1), confirming that YAP/TAZ activity is significantly upregulated in both types of human liver cancers.

\section{TAZ induces proliferation and de-differentiation of hepatocytes}

Given that YAP/TAZ activity was significantly upregulated in liver cancers, we tested whether YAP or TAZ was capable of inducing liver cancer in mice using a liver-specific transgenic approach (Ju et al. 2013). Transposons expressing a constitutively active form of human YAP (YAP ${ }^{\text {127A }}$ ) or TAZ (TAZ ${ }^{\text {S89A }}$ ) were hydrodynamically delivered to the liver together with plasmids encoding the Sleeping Beauty transposase (Fig. 2A). Expression of either YAPS127A or TAZ ${ }^{\mathrm{S} 89 \mathrm{~A}}$ in the liver did not induce visible tumors when examined at 16 weeks following the injection (Fig. 2B). Notably, microscopic examination of liver tissues from $T A Z^{S 89 A}$ mice revealed small hyperplastic lesions, which are much less frequently found in livers from mice expressing YAPS127A (Fig. 2C). Cells in the preneoplastic lesions expressing TAZ ${ }^{\text {S89A }}$ stained positive for CK19, a molecular marker for liver progenitor cells (Fig. 2C). As TAZ induced proliferation and presumably de-differentiation of hepatic cells more efficiently than YAP, we focused on TAZ for the rest of the study. 


\section{EGFR/HER2 signaling as a putative oncogenic collaborator of TAZ in liver}

Because $T A Z^{S 89 A}$ alone was insufficient to induce liver cancer in vivo, we speculated that an oncogenic collaborator is required for TAZ-mediated liver carcinogenesis in adult livers. To identify signaling pathways that are closely associated with TAZ in human carcinogenesis, we took advantage of the sensitivity analyses of compounds in human cancer cell lines (CCLs) available online through the Broad Institute (http://www.broadinstitute.org). We conducted a correlation-based analysis of 860 human CCLs with 481 compounds through the cancer therapeutic response portal (CTRP v2). The analysis identified lapatinib, erlotinib, and afatinib as the most lethal compounds for cell lines with a high copy number of the TAZ gene (Fig. 3A). All three chemical compounds are inhibitors of receptor tyrosine kinases (RTKs), mainly targeting EGFR and HER2. As the data showed that cancer cells with a high copy number of the TAZ gene were sensitive to EGFR/HER2 blockade, we speculated that the EGFR/HER2 signaling pathways may be required for the survival of cancer cells with high TAZ activity, or possibly that activation of EGFR/HER2 signaling may be required for TAZ-mediated carcinogenesis.

To confirm the association of EGFR/HER2 signaling with TAZ, we performed both database and immunohistochemistry (IHC) analyses. Analysis of the gene expression omnibus (GEO) database revealed that human HCC and $\mathrm{CC}$ with high expression levels of TAZ showed enrichment of the RAS-RAFMEK-ERK and/or PI3K-AKT-mTOR signaling pathways, both of which are downstream signaling cascades of EGFR/HER2 (Fig. 3B and Supplementary Table 2). Furthermore, IHC analysis of human HCC and CC with a high YAP/TAZ activity showed a concomitant activation of the RAS-RAF-MEK-ERK and/or PI3KAKT-mTOR signaling pathways (Fig. $3 \mathrm{C}$ and Table 1). About $87 \%$ of human liver cancer with high YAP/TAZ activity (26 out of 30 cancer samples) showed concomitant activation of the downstream signaling pathways of EGFR/HER2 (Table 1). In contrast, $11 \%$ of liver cancer samples with minimal YAP/TAZ activity (one out of nine cancer samples) revealed activation of the EGFR/HER2 signaling pathways. Thus, both the database and IHC analyses indicated a strong association between TAZ and EGFR/HER2 downstream signaling pathways in human hepatocarcinogenesis.

Table 1

$\mathrm{IHC}$ positivity in human $\mathrm{HCC}$ and $\mathrm{CC}$ tissues

\begin{tabular}{|lll|}
\hline IHC results & HCC & CC \\
\hline YAP/TAZ+ & $13 / 19(68 \%)$ & $17 / 20(85 \%)$ \\
\hline pMEK1/2 + in YAP/TAZ+ & $11 / 13(85 \%)$ & $12 / 17(71 \%)$ \\
\hline pAKT + in YAP/TAZ+ & $3 / 13(23 \%)$ & $15 / 17(88 \%)$ \\
pMEK1/2- \& pAKT- in YAP/TAZ+ & $2 / 13(15 \%)$ & $2 / 17(12 \%)$ \\
YAP/TAZ- & $6 / 19(32 \%)$ & $3 / 20(15 \%)$ \\
pMEK1/2- \& pAKT- in YAP/TAZ- & $6 / 6(100 \%)$ & $2 / 3(67 \%)$ \\
\hline
\end{tabular}




\section{TAZ induces cancer through the cooperation with RAS and PI3K signaling pathways}

Based on these data, we tested whether the EGFR/HER2 downstream signaling pathways (RAS-RAF-MEKERK and PI3K-AKT-mTOR pathways) collaborated with TAZ to induce cancer in adult livers. Transposons encoding a constitutively active form of BRAF (BRAF ${ }^{\mathrm{V} 600 \mathrm{E}}$ ) were delivered to the liver together with those encoding TAZ ${ }^{589 A}$ via hydrodynamics-based in vivo transfection (Fig. 4A). Simultaneous expression of $\mathrm{TAZ}^{\mathrm{S} 89 \mathrm{~A}}$ and $\mathrm{BRAF}{ }^{\mathrm{V} 600 \mathrm{E}}$ in the liver induced liver cancer with an incidence rate of $100 \%$ at 6 weeks following the hydrodynamic injection (Fig. 4B). Likewise, co-expression of a constitutively active form of PIK3CA (PI3K ${ }^{E 545 K}$ ) with active TAZ induced liver cancer in all the mice tested, confirming that the PI3KAKT-mTOR signaling pathway also efficiently cooperates with TAZ to induce liver cancer. Microscopic examination of the liver cancers revealed that the $\mathrm{TAZ}^{\mathrm{S} 89 \mathrm{~A}}$ plus $\mathrm{BRAF}{ }^{\mathrm{V} 600 \mathrm{E}}$ tumors were $\mathrm{HCC}$-like, while the TAZ ${ }^{\text {S89A }}$ plus PI3K ${ }^{E 545 K}$ tumors resembled CC (Fig. 4C). Western blotting of protein extracts from the tumor tissues revealed elevated expression levels of Notch 1 and NF-KB in tumors induced by TAZ ${ }^{589 A}$ plus PI3K ${ }^{\mathrm{E} 545 \mathrm{~K}}$ (Supplementary Fig. 1), compared to that in normal control livers or TAZ ${ }^{\mathrm{S} 9 \mathrm{~A}}$ plus $\mathrm{BRAF}^{\mathrm{V} 600 \mathrm{E}}$. As expected, both groups of liver cancers showed overexpression of CK19, a putative progenitor cell marker, likely due to TAZ-mediated de-differentiation of liver cells (Fig. 4C).

\section{Discussion}

YAP/TAZ signaling is involved in multiple processes during carcinogenesis, including promotion of cell proliferation, induction of tissue invasion of tumor cells, and maintenance of cancer stem cells (Zanconato et al. 2016). Several oncogenic signaling pathways have been reported to crosstalk with YAP/TAZ in carcinogenesis, including the Wnt/ $\beta$-catenin and LKB1 signaling pathways (Azzolin et al. 2014; M. Kim \& Jho 2014; Mohseni et al. 2014). Notably, the involvement of YAP in KRAS-mediated neoplastic progression in pancreatic ductal adenocarcinoma (PDAC), underscores the potential crosstalk between YAP/TAZ and RAS signaling pathways in PDAC (W. Zhang et al. 2014).

The RAS-RAF-MEK-ERK signaling pathway is activated via cell surface receptors such as EGFR, HER2, and platelet-derived growth factor receptor (PDGFR). Binding of ligands to these receptors leads to the activation of the cytoplasmic tyrosine kinases that phosphorylate tyrosine residues in the cytoplasmic tails of the receptors. This event recruits the Grb2/Shc/SOS adapter complex to the plasma membrane and subsequently converts membrane-tethered GDP-bound RAS to active GTP-bound RAS (Margolis \& Skolnik 1994; Rajalingam et al. 2007). Activated RAS triggers the mitogen-activated protein kinase signaling cascade through the RAF-MEK-ERK axis. The ligand-bound receptor tyrosine kinases also trigger the PI3K-AKT-mTOR signaling axis via phosphorylation of tyrosine residues in the cytoplasmic tails and thus recruit PI3K to the phosphorylated tyrosine residues proximal to the plasma membrane (Lemmon \& Schlessinger 2010; Porter \& Vaillancourt 1998). The RAS-RAF-MEK-ERK and PI3K-AKT-mTOR signaling pathways are activated in approximately $50 \%$ of human liver cancers, implying their significant 
roles in hepatocarcinogenesis (Dimri \& Satyanarayana 2020; Javle et al. 2006; Llovet et al. 2015; Neuzillet et al. 2014; Schmitz et al. 2007).

In this study, IHC analysis showed that RAS-RAF-MEK-ERK signaling was activated in $85 \%$ of human HCC with high YAP/TAZ activity, whereas PI3K-AKT-mTOR signaling was active only in $23 \%$ of human HCC with high YAP/TAZ activity, suggesting that the RAS-RAF-MEK-ERK pathway is the major contributor to HCC development with YAP/TAZ activation (Table 1). In line with the IHC results, co-expression of $\mathrm{BRAF}^{\mathrm{V} 600 \mathrm{E}}$ and $\mathrm{TAZ}{ }^{\mathrm{S} 89 \mathrm{~A}}$ induced $\mathrm{HCC}$ in the liver (Fig. 4). Of note, activation of PI3K signaling (through the expression of $\mathrm{PI} 3 \mathrm{~K}^{\mathrm{E} 545 \mathrm{~K}}$ ) together with TAZ activation led to CC-like liver cancer, suggesting a role for PI3K-AKT-mTOR signaling in the development of cholangiocarcinoma. Tumors induced by TAZ ${ }^{\mathrm{S} 89 \mathrm{~A}}$ plus PI3K ${ }^{\text {E545K }}$ showed elevated expression of Notch1, a critical player in CC (Banales et al. 2020; Rizvi \& Gores 2013). Further studies are needed to verify the identity of the cancer. In summary, our study identified RAS-RAF-MEK-ERK and PI3K-AKT-mTOR signaling pathways as oncogenic collaborators of TAZ during liver carcinogenesis.

\section{Declarations}

\section{AUTHOR CONTRIBUTION}

SHM and SWR designed experiments, analyzed data, and wrote the manuscript. HM performed experiments. HJC contributed to data analyses. DYK and SWR supervised the research. All authors read and approved the final manuscript.

\section{FUNDING}

The research was supported by a grant from Kyung Hee University in2020( KHU-20201758awarded to SWR), and also by the National Research Foundation of Korea (NRF) grants 2019R1I1A1A01055805 (awarded to HM), 2017R1C1B2007770(awarded to DYK), and 2019R1A2C2009518(awarded to SWR) which were funded by the Korea government (MSIT).

\section{DATA AVAILABILITY}

The data that support the findings of this study are available on request from the corresponding author.

\section{CONFLICT OF INTEREST}

The authors have declared that no competing interest exists.

\section{ETHICS APPROVAL AND CONSENT TO PARTICIPATE}

All experiments using mice were approved by the Animal Policy and Welfare Committee of the Yonsei University College of Medicine (Permit Numbers: 2013-0268 and 2013-0293). Human primary HCC and CC tissues were obtained from the biobank at the Severance Hospital, Seoul, Korea. This study was approved 
by the Independent Institutional Review Board of Severance Hospital (IRB number: 4-2018-1087) and conformed to the ethical guidelines of the Declaration of Helsinki (1975).

\section{CONSENT FOR PUBLICATION}

Not applicable.

\section{References}

1. Azzolin L, Panciera T, Soligo S et al (2014) YAP/TAZ incorporation in the $\beta$-catenin destruction complex orchestrates the Wnt response. Cell 158(1):157-170

2. Banales JM, Marin JJG, Lamarca A et al (2020) Cholangiocarcinoma 2020: the next horizon in mechanisms and management. Nat Rev Gastroenterol Hepatol 17(9):557-588

3. Chen C, Wang G (2015) Mechanisms of hepatocellular carcinoma and challenges and opportunities for molecular targeted therapy. World J Hepatol 7(15):1964-1970

4. Chen Y, Wong PP, Sjeklocha L, Steer CJ, Sahin MB (2012) Mature hepatocytes exhibit unexpected plasticity by direct dedifferentiation into liver progenitor cells in culture. Hepatology 55(2):563-574

5. Dimri M, Satyanarayana A (2020) Molecular Signaling Pathways and Therapeutic Targets in Hepatocellular Carcinoma. Cancers (Basel) 12(2)

6. Dong J, Feldmann G, Huang J, Wu S, Zhang N, Comerford SA, Gayyed MF, Anders RA, Maitra A, Pan D (2007) Elucidation of a universal size-control mechanism in Drosophila and mammals. Cell 130(6):1120-1133

7. Harvey K, Tapon N (2007) The Salvador-Warts-Hippo pathway - an emerging tumour-suppressor network. Nat Rev Cancer 7(3):182-191

8. Harvey KF, Zhang X, Thomas DM (2013) The Hippo pathway and human cancer. Nat Rev Cancer 13(4):246-257

9. Hong L, Li Y, Liu Q, Chen Q, Chen L, Zhou D (2019) The Hippo Signaling Pathway in Regenerative Medicine. Methods Mol Biol 1893:353-370

10. Hyder $\mathrm{O}$, Marques $\mathrm{H}$, Pulitano $\mathrm{C}$ et al (2014) A nomogram to predict long-term survival after resection for intrahepatic cholangiocarcinoma: an Eastern and Western experience. JAMA Surg 149(5):432438

11. Javle MM, Yu J, Khoury T et al (2006) Akt expression may predict favorable prognosis in cholangiocarcinoma. J Gastroenterol Hepatol 21(11):1744-1751

12. Ju HL, Ahn SH, Kim DY, Baek S, Chung SI, Seong J, Han KH, Ro SW (2013) Investigation of oncogenic cooperation in simple liver-specific transgenic mouse models using noninvasive in vivo imaging. PLoS One 8(3):e59869

13. Jung KS, Kim SU, Ahn SH, Park YN, Kim DY, Park JY, Chon CY, Choi EH, Han KH (2011) Risk assessment of hepatitis $B$ virus-related hepatocellular carcinoma development using liver stiffness measurement (FibroScan). Hepatology 53(3):885-894 
14. Kim DY, Han KH (2018) Staging for hepatocellular carcinoma in light of tumor heterogeneity: Time to change or update? Hepatology 67(6):2076-2078

15. Kim M, Jho EH (2014) Cross-talk between Wnt/ $\beta$-catenin and Hippo signaling pathways: a brief review. BMB Rep 47(10):540-545

16. Lemmon MA, Schlessinger J (2010) Cell signaling by receptor tyrosine kinases. Cell 141(7):11171134

17. Liu-Chittenden Y, Huang B, Shim JS, Chen Q, Lee SJ, Anders RA, Liu JO, Pan D (2012) Genetic and pharmacological disruption of the TEAD-YAP complex suppresses the oncogenic activity of YAP. Genes Dev 26(12):1300-1305

18. Llovet JM, Villanueva A, Lachenmayer A, Finn RS (2015) Advances in targeted therapies for hepatocellular carcinoma in the genomic era. Nat Rev Clin Oncol 12(7):408-424

19. Llovet JM, Zucman-Rossi J, Pikarsky E, Sangro B, Schwartz M, Sherman M, Gores G (2016) Hepatocellular carcinoma. Nat Rev Dis Primers 2:16018

20. Lu L, Li Y, Kim SM et al (2010) Hippo signaling is a potent in vivo growth and tumor suppressor pathway in the mammalian liver. Proc Natl Acad Sci U S A 107(4):1437-1442

21. Margolis B, Skolnik EY (1994) Activation of Ras by receptor tyrosine kinases. J Am Soc Nephrol 5(6):1288-1299

22. Mo JS, Park HW, Guan KL (2014) The Hippo signaling pathway in stem cell biology and cancer. EMBO Rep 15(6):642-656

23. Mohseni M, Sun J, Lau A et al (2014) A genetic screen identifies an LKB1-MARK signalling axis controlling the Hippo-YAP pathway. Nat Cell Biol 16(1):108-117

24. Moon H, Ju HL, Chung SI, Cho KJ, Eun JW, Nam SW, Han KH, Calvisi DF, Ro SW (2017) Transforming Growth Factor- $\beta$ Promotes Liver Tumorigenesis in Mice via Up-regulation of Snail. Gastroenterology 153(5):1378-1391.e1376

25. Moroishi T, Hansen CG, Guan KL (2015) The emerging roles of YAP and TAZ in cancer. Nat Rev Cancer 15(2):73-79

26. Neuzillet C, Tijeras-Raballand A, de Mestier L, Cros J, Faivre S, Raymond E (2014) MEK in cancer and cancer therapy. Pharmacol Ther 141(2):160-171

27. Pan D (2007) Hippo signaling in organ size control. Genes Dev 21(8):886-897

28. Porter AC, Vaillancourt RR (1998) Tyrosine kinase receptor-activated signal transduction pathways which lead to oncogenesis. Oncogene 17(11 Reviews):1343-1352

29. Rajalingam K, Schreck R, Rapp UR, Albert S (2007) Ras oncogenes and their downstream targets. Biochim Biophys Acta 1773(8):1177-1195

30. Rees MG, Seashore-Ludlow B, Cheah JH et al (2016) Correlating chemical sensitivity and basal gene expression reveals mechanism of action. Nat Chem Biol 12(2):109-116

31. Rizvi S, Gores GJ (2013) Pathogenesis, diagnosis, and management of cholangiocarcinoma. Gastroenterology 145(6):1215-1229 
32. Schmitz KJ, Lang H, Wohlschlaeger J, Sotiropoulos GC, Reis H, Schmid KW, Baba HA (2007) AKT and ERK1/2 signaling in intrahepatic cholangiocarcinoma. World J Gastroenterol 13(48):6470-6477

33. Seashore-Ludlow B, Rees MG, Cheah JH et al (2015) Harnessing Connectivity in a Large-Scale SmallMolecule Sensitivity Dataset. Cancer Discov 5(11):1210-1223

34. Sia D, Villanueva A, Friedman SL, Llovet JM (2017) Liver Cancer Cell of Origin, Molecular Class, and Effects on Patient Prognosis. Gastroenterology 152(4):745-761

35. Song H, Mak KK, Topol L et al (2010) Mammalian Mst1 and Mst2 kinases play essential roles in organ size control and tumor suppression. Proc Natl Acad Sci U S A 107(4):1431-1436

36. Sugiura K, Mishima T, Takano S, Yoshitomi H, Furukawa K, Takayashiki T, Kuboki S, Takada M, Miyazaki M, Ohtsuka M (2019) The Expression of Yes-Associated Protein (YAP) Maintains Putative Cancer Stemness and Is Associated with Poor Prognosis in Intrahepatic Cholangiocarcinoma. Am J Pathol 189(9):1863-1877

37. Tanimizu N, Nakamura Y, Ichinohe N, Mizuguchi T, Hirata K, Mitaka T (2013) Hepatic biliary epithelial cells acquire epithelial integrity but lose plasticity to differentiate into hepatocytes in vitro during development. J Cell Sci 126(Pt 22):5239-5246

38. Villanueva A (2019) Hepatocellular Carcinoma. N Engl J Med 380(15):1450-1462

39. Xu MZ, Yao TJ, Lee NP, Ng IO, Chan YT, Zender L, Lowe SW, Poon RT, Luk JM (2009) Yes-associated protein is an independent prognostic marker in hepatocellular carcinoma. Cancer 115(19):4576-4585

40. Yim SY, Lee J-S (2019) The Genomic Landscape and Its Clinical Implications in Hepatocellular Carcinoma. J Liver Cancer 19(2):97-107

41. Yimlamai D, Christodoulou C, Galli GG, Yanger K, Pepe-Mooney B, Gurung B, Shrestha K, Cahan P, Stanger BZ, Camargo FD (2014) Hippo pathway activity influences liver cell fate. Cell 157(6):13241338

42. Zanconato F, Cordenonsi M, Piccolo S (2016) YAP/TAZ at the Roots of Cancer. Cancer Cell 29(6):783803

43. Zender L, Spector MS, Xue W et al (2006) Identification and validation of oncogenes in liver cancer using an integrative oncogenomic approach. Cell 125(7):1253-1267

44. Zhang N, Bai H, David KK, Dong J, Zheng Y, Cai J, Giovannini M, Liu P, Anders RA, Pan D (2010) The Merlin/NF2 tumor suppressor functions through the YAP oncoprotein to regulate tissue homeostasis in mammals. Dev Cell 19(1):27-38

45. Zhang W, Nandakumar N, Shi Y et al (2014) Downstream of mutant KRAS, the transcription regulator YAP is essential for neoplastic progression to pancreatic ductal adenocarcinoma. Sci Signal 7(324):ra42

\section{Figures}


A

HCC

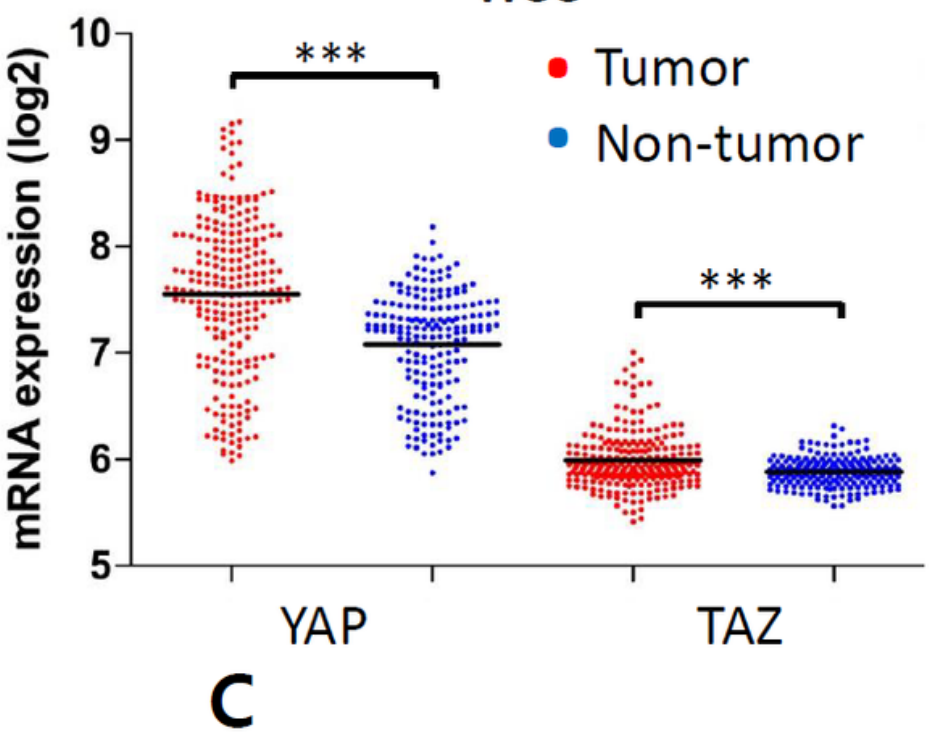

B

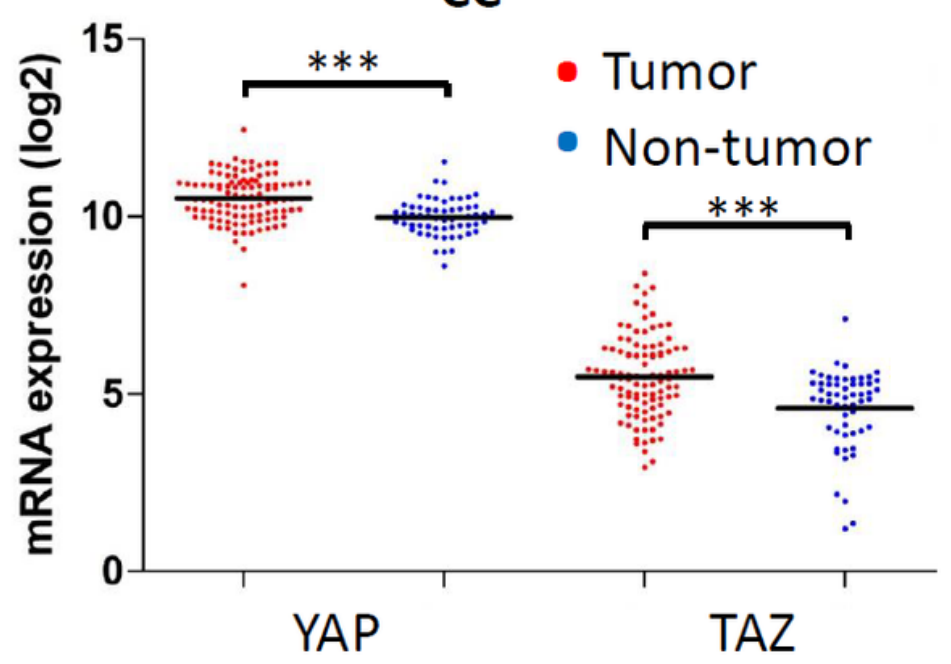

HCC

CC

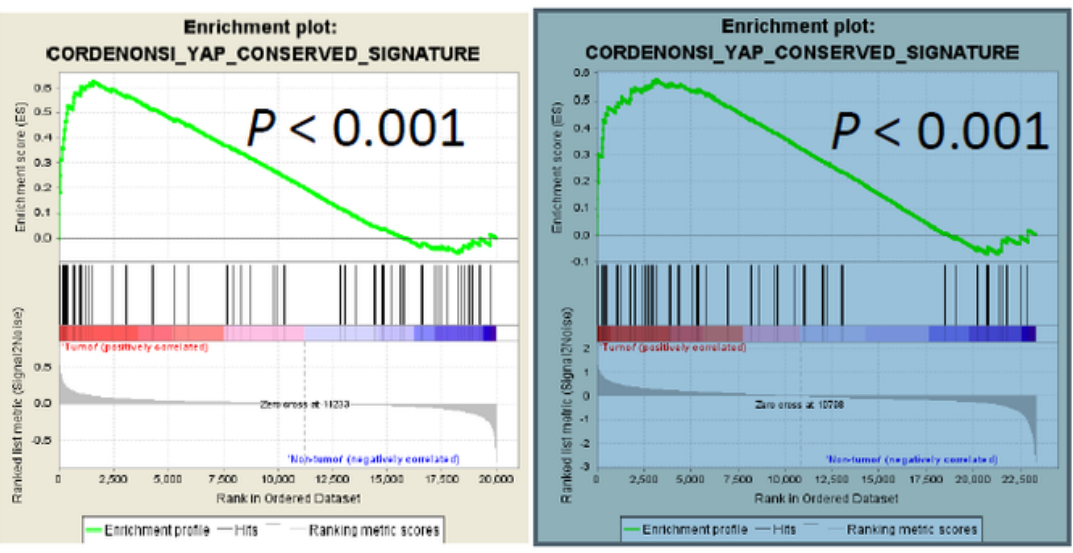

\section{Figure 1}

Activation of YAP/TAZ signaling in human liver cancers.(A, B)Expression levels of YAP and TAZ (WWTR1) in tumor and non-tumor counterparts were compared using gene expression omnibus (GEO) database for HCC (A)and CC (B). Mean expression level of each group is indicated with horizontal lines. ( $\left.{ }^{* \star *}, P<0.001\right)$. (C)Enrichment plots from gene set enrichment analysis (GSEA). YAP/TAZsignalsare highly enriched both in $\mathrm{HCC}$ and $\mathrm{CC}(\mathrm{P}<0.001)$. Barcode indicates gene positions and the $y$-axis indicates the extent of enrichment. 
A

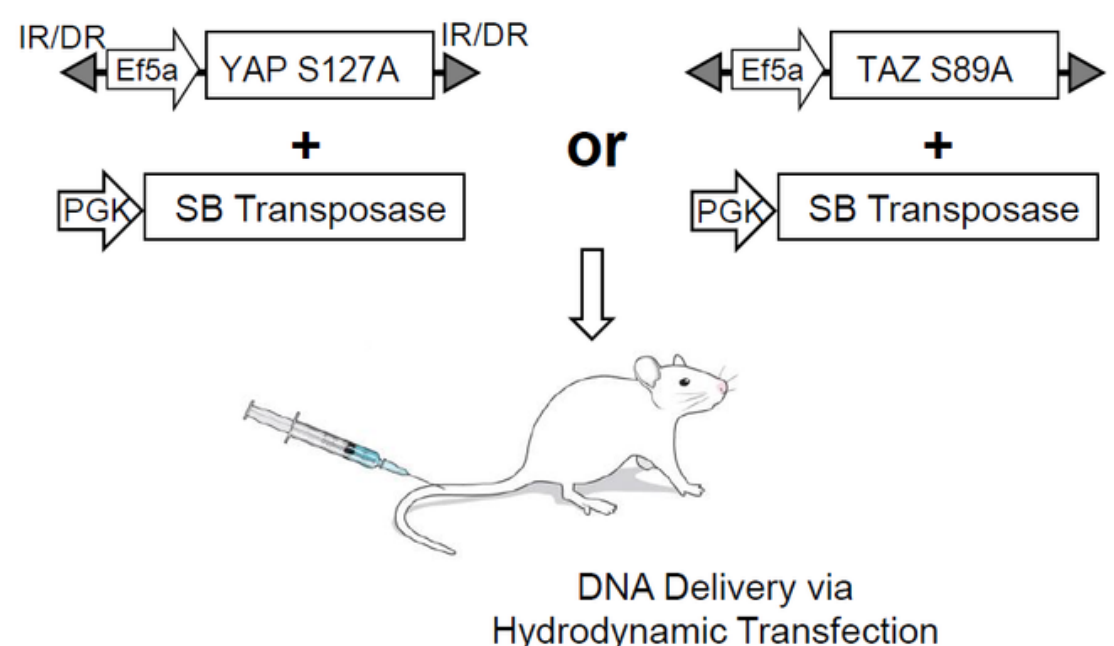

B

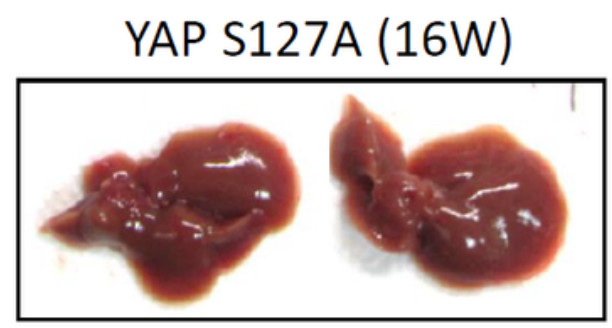

TAZ S89A (16W)

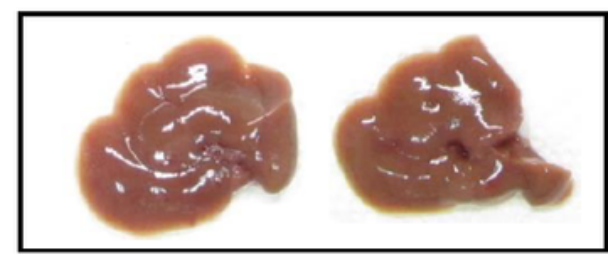

C

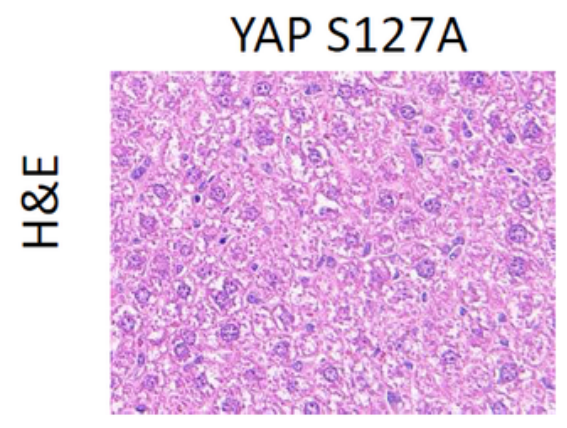

TAZ S89A
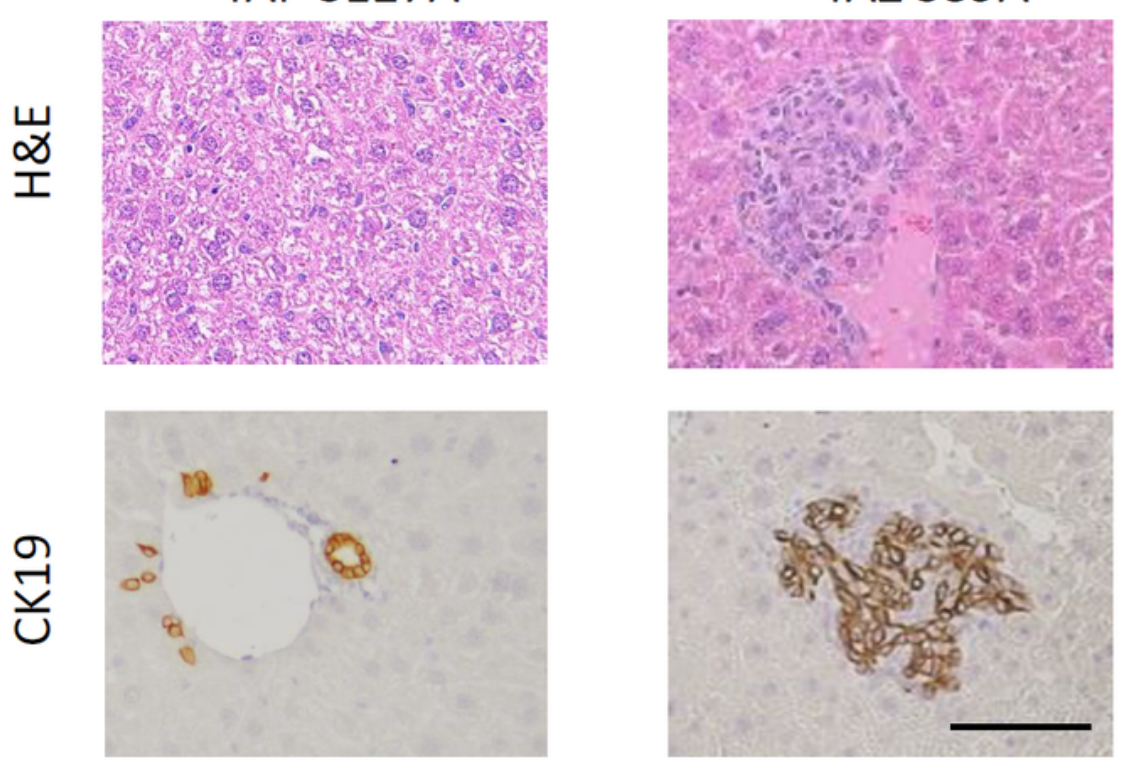

\section{Figure 2}

YAP or TAZ alone does not induce tumors. (A) Schematic illustration of the experimental procedure. Transposons encoding each oncogene were mixed with plasmids expressing the Sleeping Beauty transposase and injected hydrodynamically(B)Gross morphology of representative liversexpressingYAPS127 and TAZS89A, respectively, are shown. Livers were harvested at 16 weeks following thehydrodynamic injection $(n=5)$. (C)Microscopic images showing H\&E and IHC staining for 
CK19 in sections of livers shown in (B). In sections of livers expressingYAPS127, ductal cells positively stained for CK19 are shown. Scale bar, $50 \mu \mathrm{m}$.

A

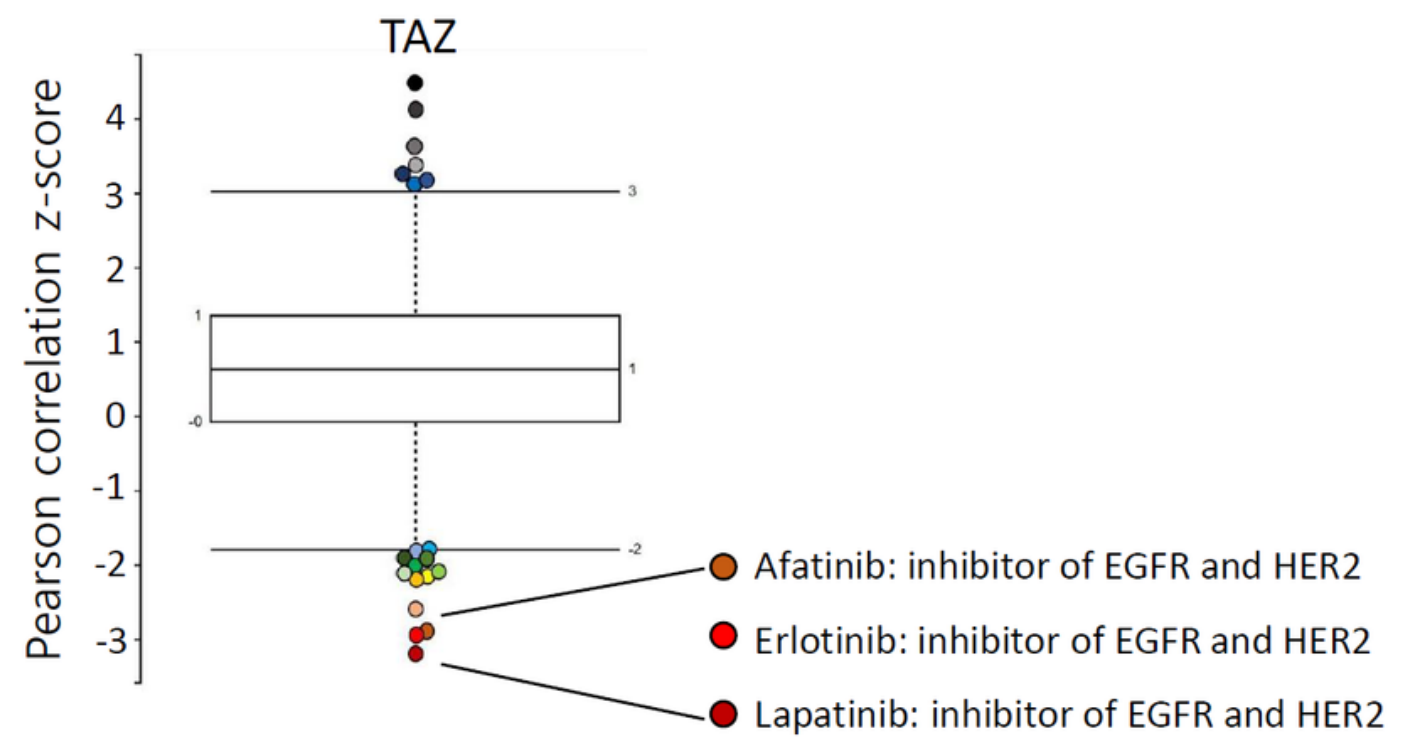

B
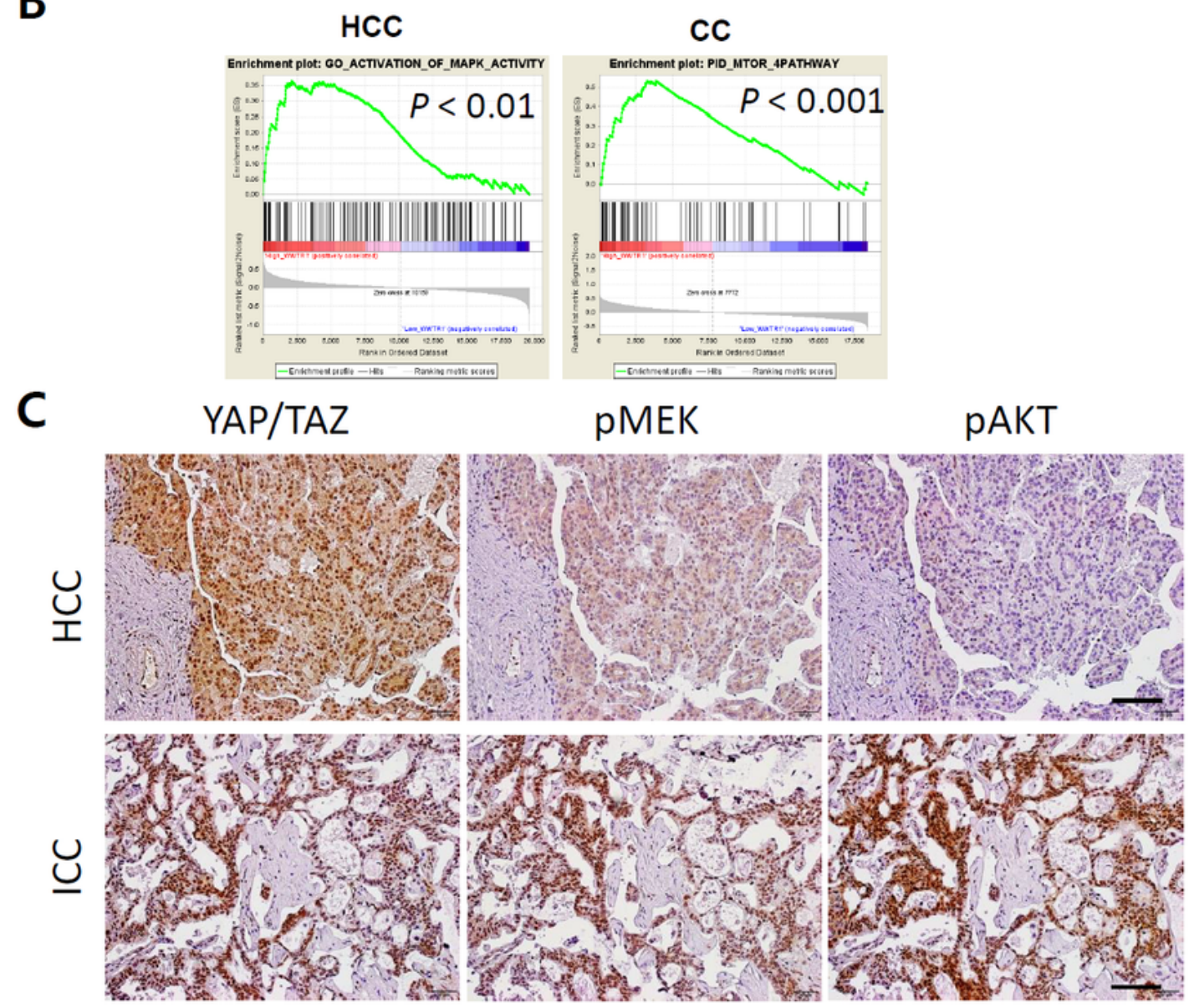

Figure 3

EGFR/HER2 signaling pathways are associated with TAZ in human cancer.(A)Correlation between chemical sensitivity and copynumber ofTAZ gene. Y-axis indicates Z-scored Pearson correlations. Box plot represents Tukey outliers.(B)GSEA was performed withregard to MAPK and mTOR signaling 
pathwaysusingHCC (GSE54236) and CC (GSE32225) wth high and low levels of TAZ expression. (C) IHC analyses of YAP/TAZ, pMEK, and pAKTin human HCC and CC.Scale bar, $100 \mu \mathrm{m}$.
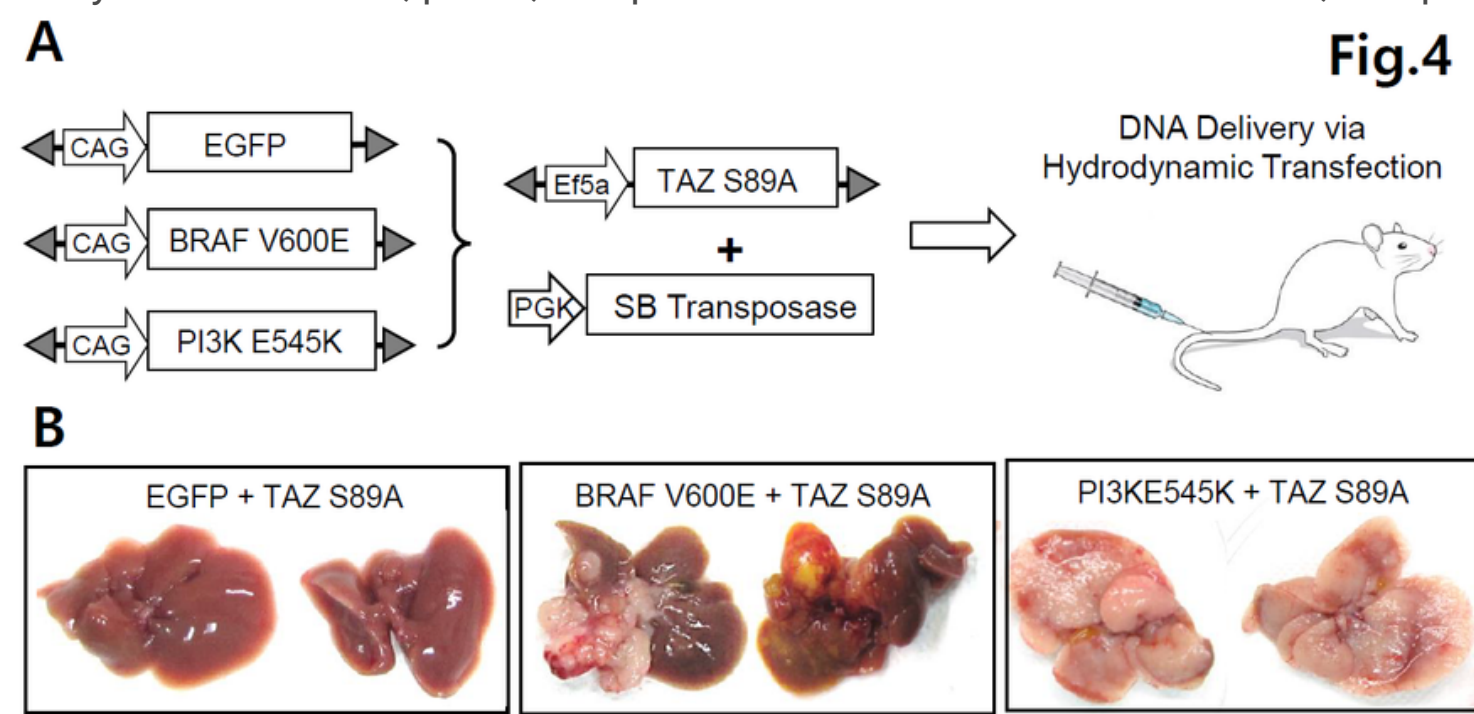

DNA Delivery via Hydrodynamic Transfection
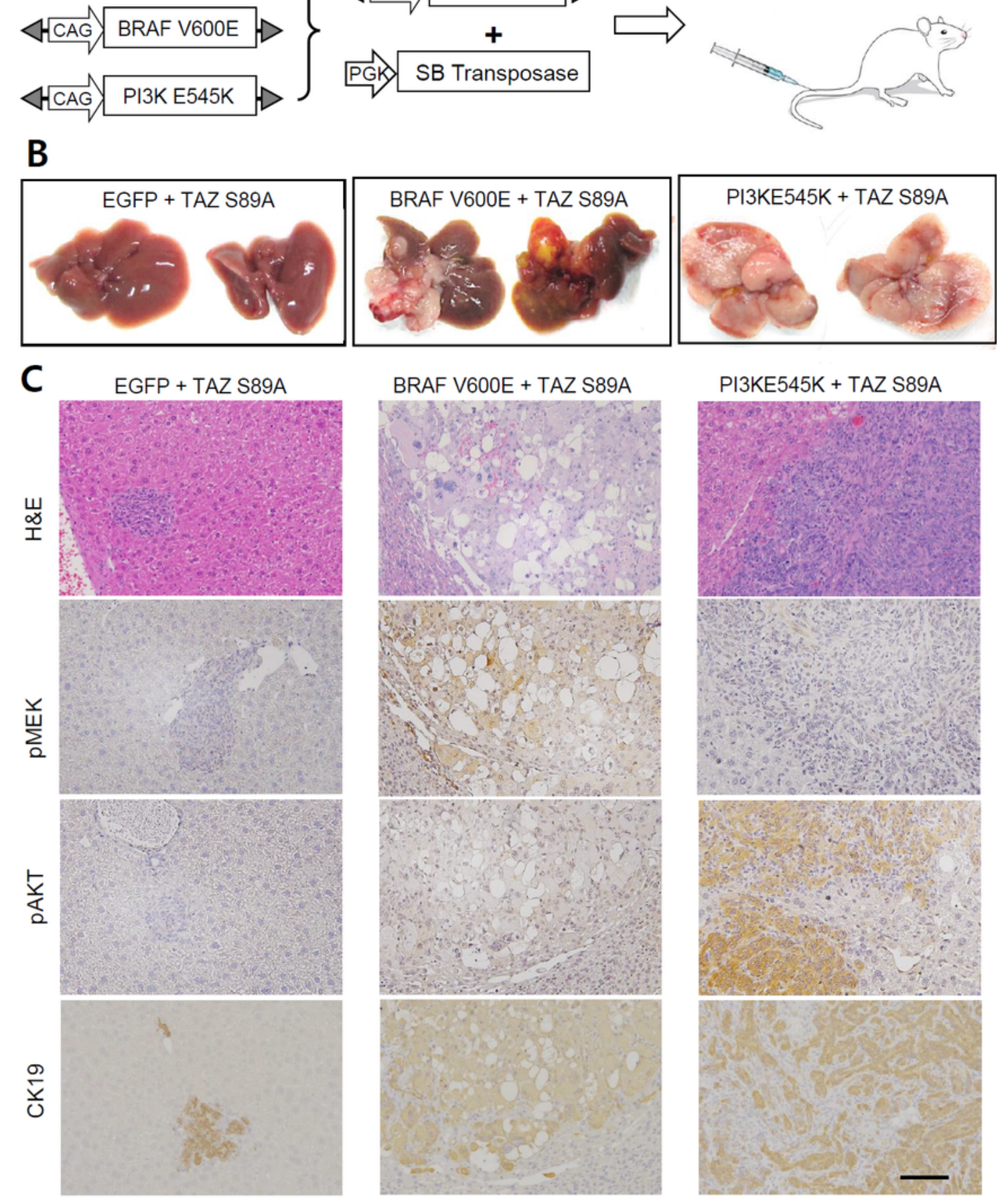

\section{Figure 4}

TAZS89A induces liver cancer incollaboration with either BRAFV600E or PI3KE545K.(A)Schematic illustration of the experimental procedure. (B)Gross morphology of representative livers expressing EGFP (control), BRAFV600E, and PI3KE545K, respectively, together with TAZS89A. Livers were harvested at 6 
weeks following the hydrodynamic injection( $\mathrm{n}=5 \mathrm{mice} / \mathrm{group})$.(C)H\&E and IHC staining of tumor sections from mice expressing EGFP, BRAFV600E, and PI3KE545K, respectively, together with TAZS89A. Scale bar, $100 \mu \mathrm{m}$.

\section{Supplementary Files}

This is a list of supplementary files associated with this preprint. Click to download.

- SUPPLEMENTARYTABLES.docX

- SupplemetaryFigure.pdf 